\title{
Tidal Field Numerical Simulation of Hulushan Bay
}

\author{
Yuchi Niu, Jianguo Lin and Lei Liu \\ Department of Environmental Science and Engineering, Dalian Maritime University, China
}

\begin{abstract}
With the development of marine economy in China in recent years, The industrial zone of Dalian Changxing island has been upgraded to a national economic and technological development zone in 2010, not only take on a new mission and task, but also the tidal field characteristics study of Changxing island proposed new requirements. This paper builds a two-dimensional tidal current mathematical model of Changxing island, and compared the simulation results with measured data. In results, simulation agrees well with the measured degree, this models can be more truly reflect the characteristics of the target sea area, It can provide the basic data for the construction of the sea, environmental protection.
\end{abstract}

Keywords-changxing island; hulushan bay; numerical Simulation

\section{INTRODUCTION}

Changxing Island is located in the west of Liaodong Peninsula, Dalian's The Bohai Sea side of the middle coastline. It is the largest island in the north of the Yangtze River, the geographical coordinates of longitude $121^{\circ} 16^{\prime}$, latitude $39^{\circ}$ 31'. Changxing Island as an important port of Dalian is located in the Bohai Sea; its geographical position is extremely superior. The Hulushan bay mouth to the west, north-south width of $10 \mathrm{~km}$, and water depth of about $20 \mathrm{~m}$, its north and south sides were Xizhong Island and Changxing Island. The bottom of the bay is an underwater shoals, water depth from $0 \mathrm{~m}$ to $2 \mathrm{~m}$, slope from $1.8 \times 10^{-3}$ to $2.0 \times 10^{-3}$, tilt outward from the bay sea.

With the development of Liaoning coastal economic belt, Changxing Island waters will build more than sea-related projects in the future. But in fact, long sequence of measured data is difficult to obtain, therefore, the numerical simulation of target sea area can provide data for sea-related projects to make up for lack of measured data, but also can provide good technical support to calculate capacity of ocean[1] .

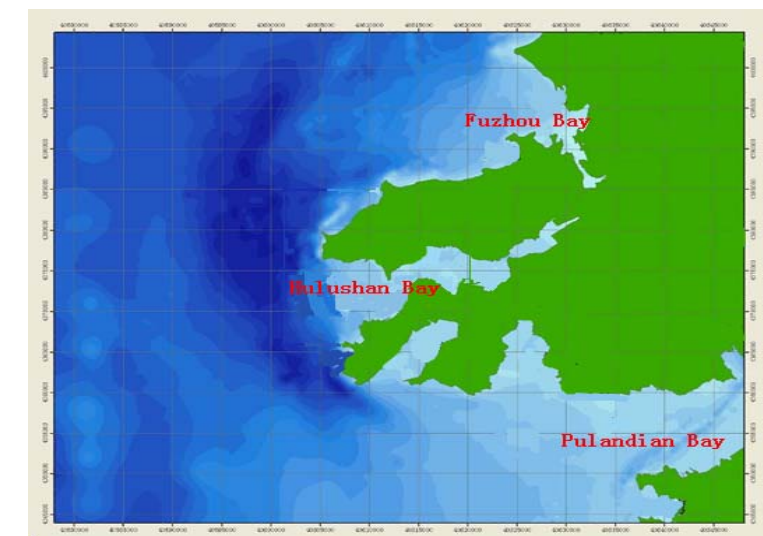

FIGURE I. SCHEMATIC DIAGRAM OF CHANGXING ISLAND WATERS.

\section{TIDAL FLOW CONTROL MODEL}

\section{A. Governing Equation}

Integration of the horizontal momentum equations and the continuity equation over depth $h=\eta+d$ the following two-dimensional shallow water equations are obtained [2]:

$$
\frac{\partial h}{\partial t}+\frac{\partial h \bar{u}}{\partial x}+\frac{\partial h \bar{v}}{\partial y}=h S
$$

$\frac{\partial h \bar{u}}{\partial t}+\frac{\partial h \bar{u}^{2}}{\partial x}+\frac{\partial h \overline{v u}}{\partial y}=$

$f \bar{v} h-g h \frac{\partial \eta}{\partial x}-\frac{g h^{2}}{2 \rho_{0}} \frac{\partial \rho}{\partial x}+\frac{\tau_{s x}}{\rho_{0}}-\frac{\tau_{b x}}{\rho_{0}}+\frac{\partial}{\partial x}\left(h T_{x x}\right)+\frac{\partial}{\partial y}\left(h T_{x y}\right)+h u_{s} S$

$\frac{\partial h \bar{v}}{\partial t}+\frac{\partial h \overline{v u}}{\partial x}+\frac{\partial h \bar{v}^{2}}{\partial y}=$

$-f \bar{u} h-g h \frac{\partial \eta}{\partial y}-\frac{g h^{2}}{2 \rho_{0}} \frac{\partial \rho}{\partial y}+\frac{\tau_{s y}}{\rho_{0}}-\frac{\tau_{b y}}{\rho_{0}}+\frac{\partial}{\partial x}\left(h T_{x y}\right)+\frac{\partial}{\partial y}\left(h T_{y y}\right)+h v_{s} S$

where $t$ is the time; $x, y$ and $z$ are the Cartesian co-ordinates; $\eta$ is the surface elevation, ${ }_{d}$ is the still water depth; $h=\eta+d$ is the total water depth; $u, v$ and $w$ are the velocity components in the $x, y$ and $z$ direction; $f$ is the Coriolis parameter; $g$ is the gravitational acceleration; $\rho$ is the density of water; $S$ is the magnitude of the discharge due to point sources $S$; $u_{s}$ and $v_{s}$ is the velocity by which the water is discharged into the ambient water.

\section{B. Numerical Solution}

The discretization in solution domain is performed using a finite volume method. The spatial domain is discretized by subdivision of the continuum into non-overlapping elements. In the two-dimensional case the elements can be arbitrarily shaped polygons, however, here only triangles and quadrilateral elements are considered. In this paper, using triangular mesh model and local encryption. For the time integration, we can choose low order calculation method in ensuring the accuracy of the case [3].

\section{Mesh Generation}

In order to ensure the local tidal field calculation conform to the trend of the field of physical characteristics as whole, the paper based on the large and small model in nested way to calculate. The large model contains the whole the Bohai Sea, small model longest east-west is about $100 \mathrm{~km}$ and longest north- south is about $80 \mathrm{~km}$. Figure 2,figure 3 were given computing grid of large and small. The paper based on the triangular grid can fitting shoreline and building complex boundary. Large model has 9030 mesh nodes, small model has 
27284 mesh nodes, The maximum length of grid space step is $5000 \mathrm{~m}$, and the minimum is $7 \mathrm{~m}$.

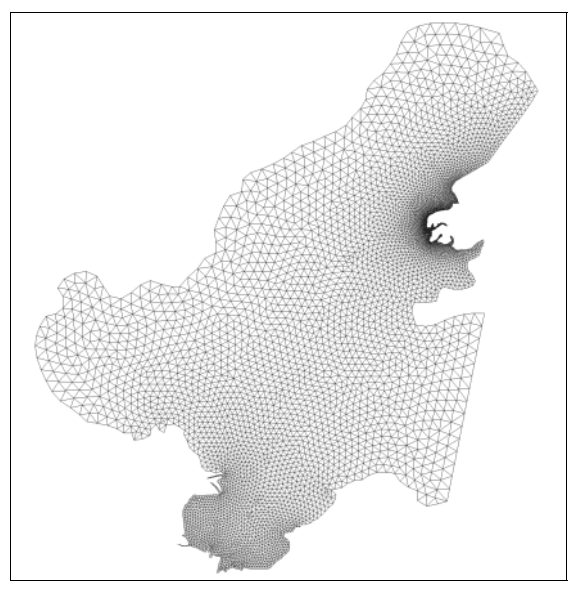

FIGURE II. SCHEMATIC DIAGRAM OF LARGE MODEL GRID.

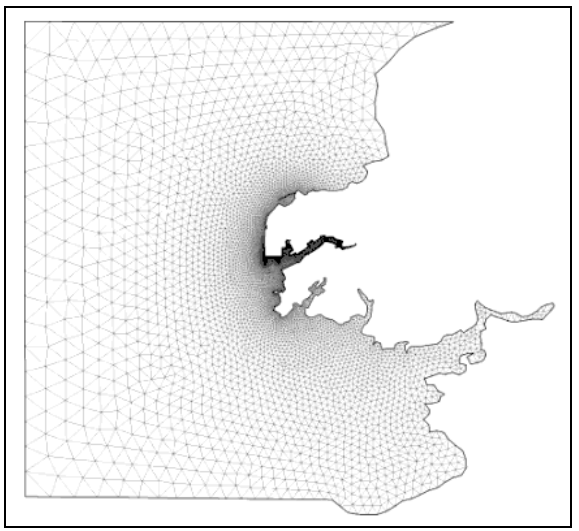

FIGURE III.

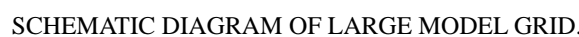

\section{Boundary Conditions}

(1)open boundary :The open boundary conditions can be specified either in form of a unit discharge or as the surface elevation for the hydrodynamic equations. For transport equations either a specified value or a specified gradient can be given. For this numerical simulation, use the tide level predicted by The Bohai Sea tide prediction system as a preliminary calculation of open borders.

(2)Closed boundary: Along closed boundaries (land boundaries) normal fluxes are forced to zero for all variables. For the momentum equations this leads to full-slip along land boundaries.

(3)Flood and Dry: This paper used the model of the default flood and wet boundary treatment method. When the water depth is less than the wetting depth the problem is reformulated and only if the water depth is less than the drying depth the element/cell is removed from the simulation. The flooding depth is used to determine when an element is flooded. The reformulation is made by setting the momentum fluxes to zero and only taking the mass fluxes into consideration [4].

\section{EXAMPLES OF APPLICATION}

\section{A. The Establishment of Model}

On the basis of the collected relevant historical data, and combined with the feature of the ocean current and tide, to simulate the tidal field of the target area and generates a two-dimensional tidal model schematic diagram of Hulushan Bay. As shown in Figure 4 and 5.

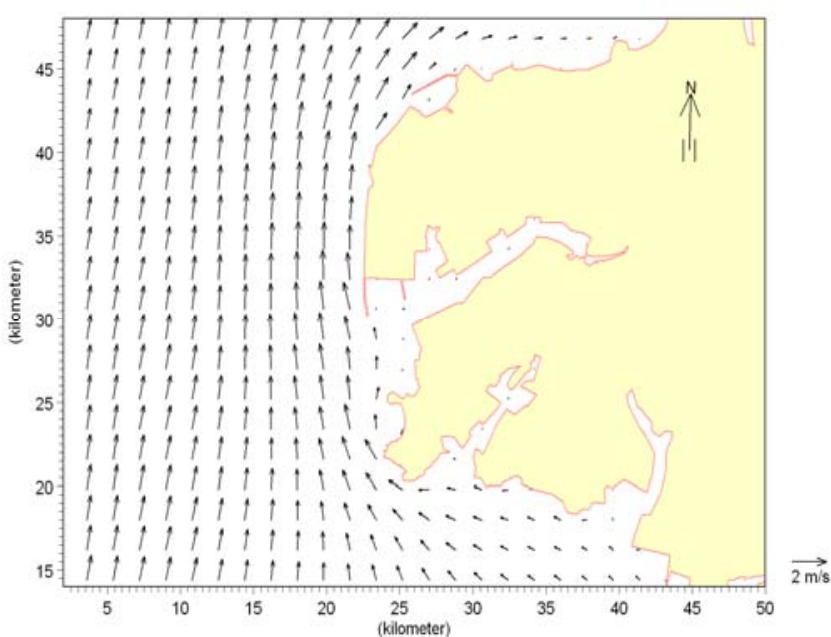

FIGURE IV. SCHEMATIC DIAGRAM OF FLOOD MIDDLE TIDAL FIELD.

\section{B. Model Validation}

In the paper, the data of water level, flow velocity and flow direction is measured by 08:00 on May 8th, 2012 to 12:00 on the 9th. Figure 6 to 8 is the comparison between measured and calculated values from each tide level station. (The 1\# tide level station is located in the bay. Due to circulation affected, the velocity and direction of flow of some tide level station is more complex).

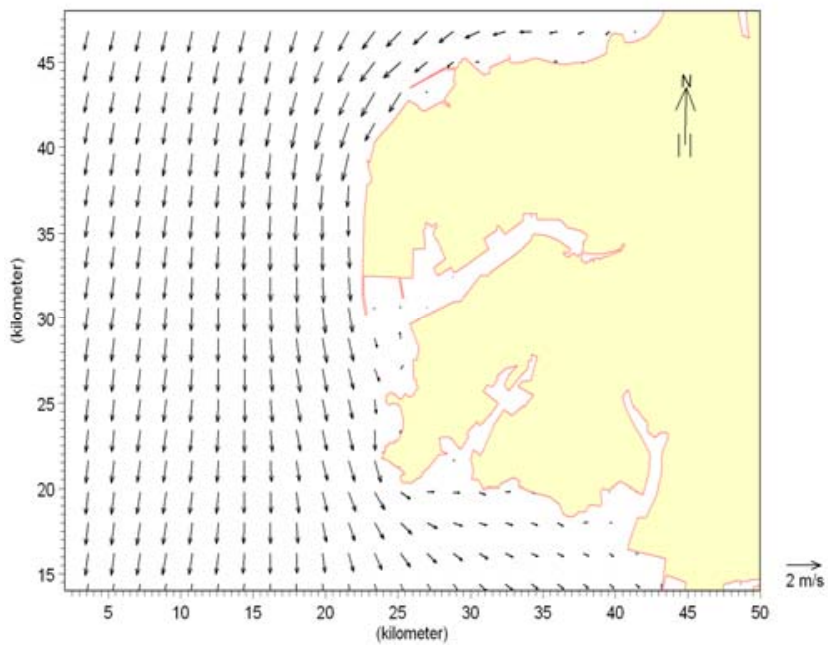

FIGURE V. SCHEMATIC DIAGRAM OF EBB MIDDLE TIDAL FIELD

In the results, simulation agrees well with the measured degree. Therefore, established mathematical model of this 
paper is feasible; this model can be more truly reflect the tidal motion feature of the Hulushan bay.

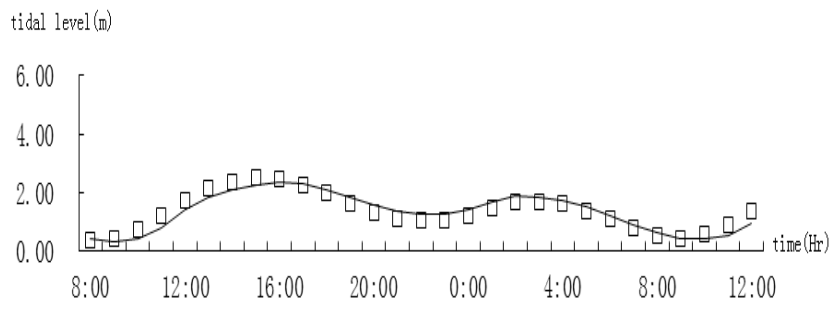

FIGURE VI. VERIFICATION RESULTS OF BACHA TIDE LEVEL STATION.
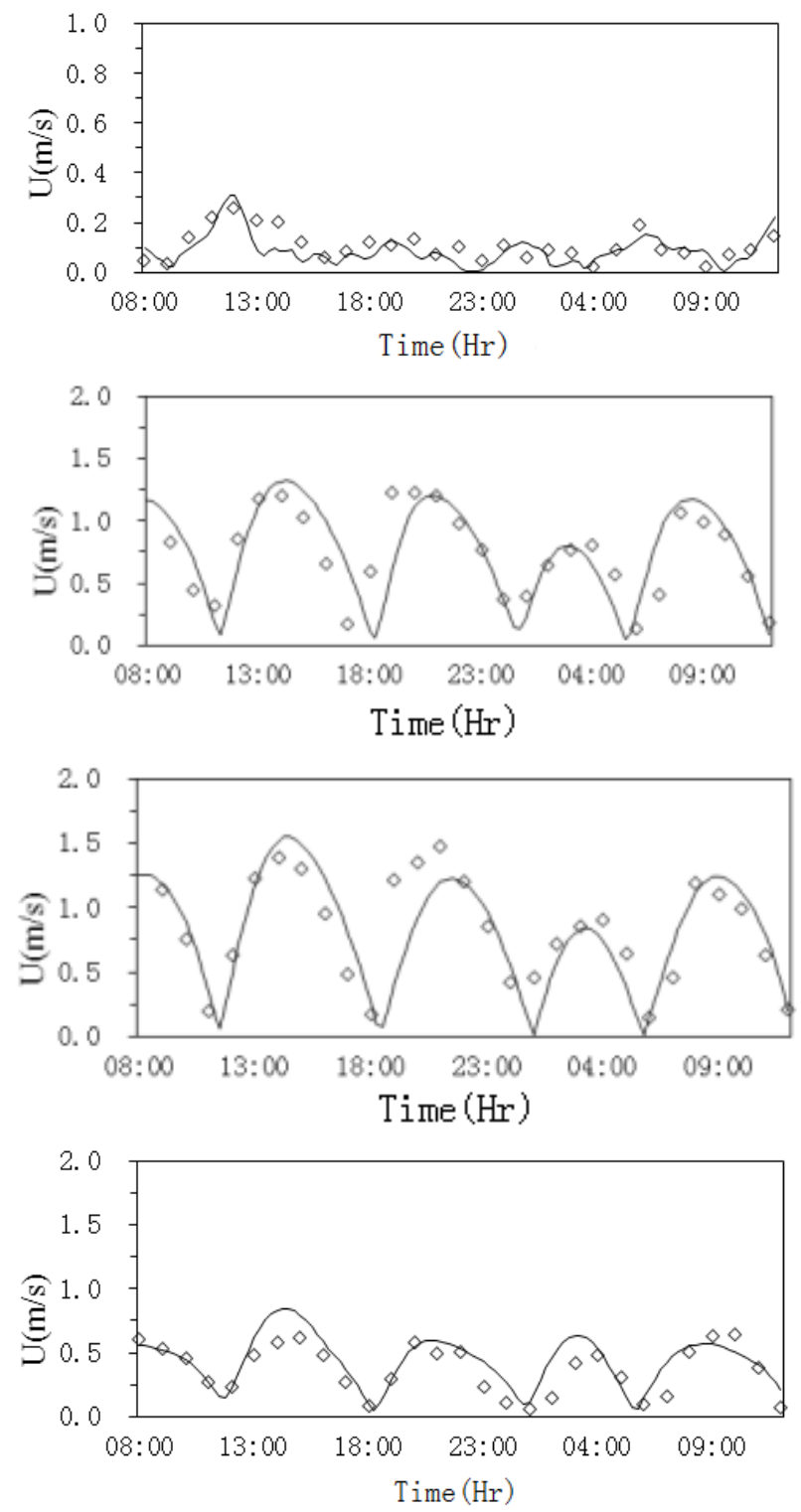

FIGURE VII. THE VERIFICATION RESULTS OF TIDAL FLOW VELOCITY.
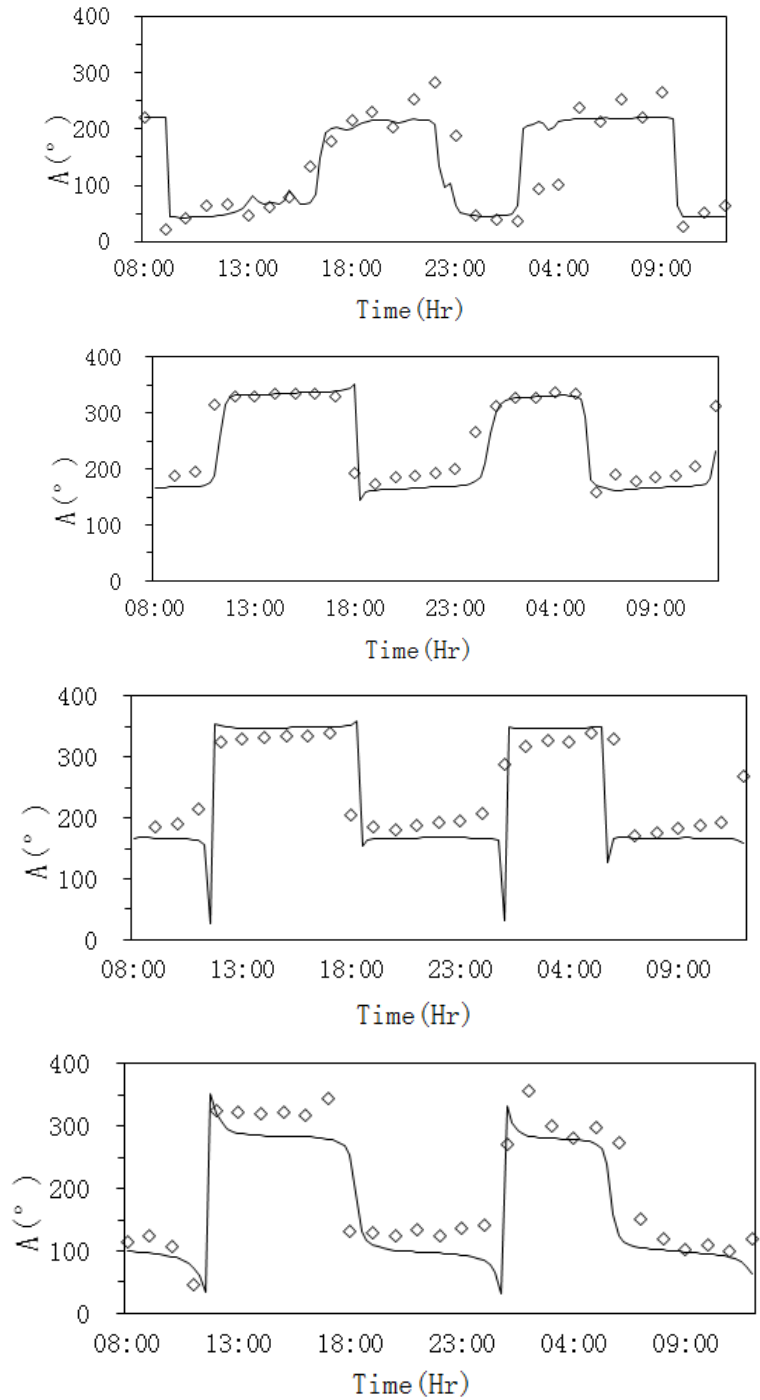

FIGURE VIII. THE VERIFICATION RESULTS OF TIDAL FLOW DIRECTION.

\section{Model Result Analysis}

(1)Tide movement form: The trend of target area reciprocating movement c along the shoreline substantially, high tide to approximate N, ebb tide to approximate S. Since the construction of the west breakwater, north breakwater and the temporary cofferdam of the south bank. Forming a circulation zone in the inside of the Hulushan bay when high tide and low tide. The flow direction of hulushan tip in ENE or WSW substantially

(2)Tide change process: the Slack water of each tide stations occurs in the high, ebb tide generally. The maximum velocity generally occurs in half tide. Affected Fuzhou Bay and Pulandian Bay, Tide duration of high and ebb tide is different in different waters. The ebb tidal duration of the Fuzhou Bay and the Pulandian Bay is longer than flood tide, on the contrary in the Hulushan Bay.

(3)Tide intensity distribution: The characteristics of tidal distribution are that offshore waters velocity is high and Bay 
waters velocity is low. The Hulushan bay average flow velocity between $0.09 \sim 0.14 \mathrm{~m} / \mathrm{s}$. Offshore waters average flow velocity between $0.69 \sim 0.90 \mathrm{~m} / \mathrm{s}$.

\section{CONCLUSION}

In this paper, the author builds a two-dimensional tidal current mathematical model of Changxing Island. Not only through Encryption Grid to flexible processing complex terrain, but also can greatly reduce operation time.

the tidal field of this model is consistent with the natural condition analysis results and the previous research results, we can think that the tidal field can be more truly reflect the characteristics of the target sea area, It can provide the necessary hydrodynamic conditions for the sediment movement simulation and sea waves simulation.

\section{REFERENCES}

[1] Assessment Center of Environmental Engineering of EPD. Technical Methods of Environmental Impact Assessment [M].Beijing: China Environmental Science Press, 2012.

[2] Wang Q G, Dai. Numerical Model of Thermal Discharge from Laibin Power Plant Based on Mike21FM [J].Research of Environmental Sciences2009,22(3):332-336.

[3] Li N,Ye M.COD Diffusion Simulation Based on MIKE21 in Sewage Outfall of Peiling Section of three Gorges Reservoir Area and Its Impact on Downstream Water Quality[J].Journal of North China Institute of Water Conservancy and Hydroelectric Power,2011(1):128-131.

[4] Wang L Y. The Numerical Simulation of 1-D and 2-D River on MIKE21 FM [D].Dalian: Dalian University of Technology, 2007. 